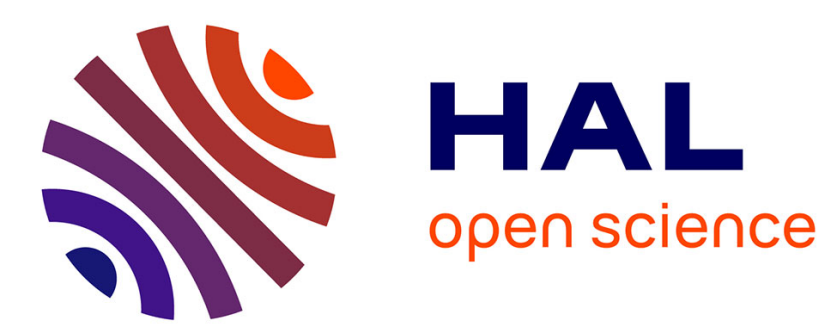

\title{
Organogel formation rationalized by Hansen solubility parameters: improved methodology
}

Danilo Rosa Nunes, Matthieu Raynal, Benjamin Isare, Pierre-Antoine Albouy, Laurent Bouteiller

\section{- To cite this version:}

Danilo Rosa Nunes, Matthieu Raynal, Benjamin Isare, Pierre-Antoine Albouy, Laurent Bouteiller. Organogel formation rationalized by Hansen solubility parameters: improved methodology. Soft Matter, 2018, 14 (23), pp.4805-4809. 10.1039/c8sm00562a . hal-02357562

\section{HAL Id: hal-02357562 \\ https://hal.science/hal-02357562}

Submitted on 10 Nov 2019

HAL is a multi-disciplinary open access archive for the deposit and dissemination of scientific research documents, whether they are published or not. The documents may come from teaching and research institutions in France or abroad, or from public or private research centers.
L'archive ouverte pluridisciplinaire HAL, est destinée au dépôt et à la diffusion de documents scientifiques de niveau recherche, publiés ou non, émanant des établissements d'enseignement et de recherche français ou étrangers, des laboratoires publics ou privés. 


\title{
Organogel formation rationalized by Hansen solubility parameters: improved methodology
}

\author{
Danilo Rosa Nunes, ${ }^{\text {ab }}$ Matthieu Raynal, ${ }^{a}$ Benjamin Isare, ${ }^{a}$ Pierre - Antoine Albouy ${ }^{b}$ and Laurent Bouteiller ${ }^{* a}$ \\ An organogel is obtained when a low molar mass compound forms a network of anisotropic fibres in a liquid that is therefore transformed \\ into a macroscopic solid. Various approaches have been proposed to correlate organogel formation and Hansen solubility parameters. These \\ approaches are well adapted to specific experimental datasets but lack universality. A general method to determine the gelation domain from \\ the solubility data of low molecular weight gelators is here reported.
}

\section{Introduction}

Organogels are formed by the dissolution at high temperatures of a small amount (usually $<2$ wt\%) of a low molecular weight gelator (LMWG) in a liquid. Upon cooling, the LMWGs tend to self-assemble preferentially in a uni-directional way leading to the formation of fibres, strands, or tapes. These structures trap the liquid molecules resulting in solid-like materials. ${ }^{1-4}$ Although the interest in organogels has seen an increase in the last years $^{5-8}$ the discovery of new LMWGs is still mainly the result of serendipity and their gelation abilities are usually probed by exhaustive trial and error processes. With a long term aim of avoiding this laborious work, we proposed a methodology for organogel rationalization based on Hansen Solubility Parameters (HSP).9,10 HSP result from a thermodynamic approach and allow to predict, in particular, the solubility of polymers. ${ }^{11-13}$ According to this approach, each molecule has a set of three different cohesive energy densities due to Dispersive interactions $\left(\delta_{D}\right)$, Polar interactions $\left(\delta_{P}\right)$ and Hydrogen bonds $\left(\delta_{H}\right)$, that can then be used as coordinates to plot a 3D diagram (Hansen space). By testing the solubility of a given compound in a set of liquids with known HSP, a sphere can be computed in the Hansen space, so that this sphere contains most of the liquids that dissolve the tested compound. If an untested liquid has HSP values positioned inside the solubility sphere, then this liquid is predicted to dissolve the compound.

We previously showed that when such solubility tests are performed on a LMWG it is possible to determine a solubility sphere, but also a gelation sphere, that contains most of the liquids that are gelled.9,10 The correlation of HSP with the gelation ability of LMWGs has since then been applied in numerous publications, with varied approaches of the data treatment and presentation. ${ }^{14}$ Some studies performed a simple qualitative description of the Hansen space (without determining a gelation sphere) or used a 1D or 2D projection of Hansen space (Teas Plot) to simplify the data treatment and representation. ${ }^{15-29}$ More interestingly, some studies proposed alternative procedures to determine a gelation domain from their specific experimental data. ${ }^{30-38}$ While these modifications of the data treatment are certainly well suited to particular datasets, the natural question that arises is whether they are of general applicability.
Moreover, to study how the gelation domain of various LMWGs evolves with the structure (within a set of related LMWGs, as attempted in a previous publication ${ }^{39}$ ), a universal methodology is needed so that studies from various research groups can be quantitatively compared. With this we would be able to trace if there is a general relationship between LMWG structure and gelation domain in the Hansen space.

Therefore, this publication presents a revision of our initial methodology, with the necessary changes to account for the new data and to allow an easy comparison between different experimental studies.

\section{Results and discussion}

To correlate the gelation of a LMWG with HSP the first step is to collect a consistent dataset. The details have been discussed previously, ${ }^{10}$ and we simply recall here that all gelation tests have to be performed in identical conditions (e.g. concentration, dissolution temperature, cooling rate) and in a range of liquids widely distributed in Hansen space. The results are then plotted as points in the Hansen space and grouped in three categories that correspond to liquids that are gelled by the LMWG (G), liquids that dissolve the LMWG (S) and liquids in which the LMWG is insoluble or precipitates (P). We now focus our attention on the determination of the gelation sphere.

\section{The initial method}

The accepted methodology to determine the solubility sphere of any kind of solute is to find the centre and radius of a sphere so that most $\mathbf{S}$ points should lie inside the sphere and most other points ( $\mathbf{P}$ and $\mathbf{G}$ in the case of a LMWG) should lie outside. ${ }^{11-13}$ By analogy, in our previous work, ${ }^{9,10}$ the centre and radius of the gelation sphere were determined in such a way that most $\mathbf{G}$ points should lie inside the sphere and most $\mathbf{S}$ and $\mathbf{P}$ points should lie outside. The consequence of this procedure is that no or very little overlap of the gelation and solubility spheres is expected. We call this procedure NO (for No Overlap). The solubility and gelation spheres obtained with this NO procedure presented a good description of the data of several LMWGs available in the literature..$^{9,10}$ The same methodology was also successfully used in a comprehensive gelation study of amide derivatives of (R)-12-hydroxystearic acid gelators. ${ }^{39}$ 


\section{Do gelation and solubility spheres share the same centre?}

Since we first published our approach, other methods to determine the gelation sphere have appeared. In particular, Weiss et $\mathrm{al}^{35,36}$ proposed an approach that resulted in concentric spheres (CS), with the solubility sphere being enclosed by the gelation sphere. This was achieved by taking into account the centre of the solubility sphere and force fitting the gelation sphere to have the same centre. This CS method presented a good fit in the Hansen space of some LMWGs and made less complex the data interpretation. ${ }^{35,36}$ However, it was proven to be not general, since Weiss and Rogers et al in later studies $^{30,32,33,37,38}$ showed several cases where this CS treatment is not the best alternative. Indeed, there are LMWG where the G points are not uniformly distributed around the solubility sphere in Hansen space. To clearly exemplify this lack of generality, we try to apply the CS method to the data obtained in our previous study, using the LMWG C4 ${ }^{39}$ (Fig. 1).
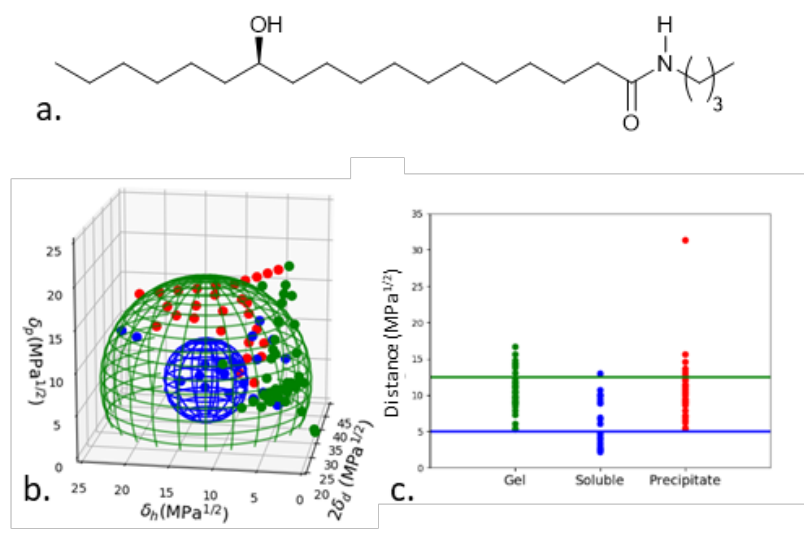

Figure 1. a) LMWG C4 b) Data for C4 at $2 \mathrm{wt} / \mathrm{v} \%$ represented in Hansen space. Liquids are represented by full circles and calculated domains are represented by meshed spheres. Green: gel; Red: precipitate. Blue: soluble. The solubility and gelation spheres are forced to have the same centre (CS method). c) Distances in HSP space of the liquids tested to the centre of the gelation and solubility sphere, highlighting the radius of the spheres by a green (gelation) or blue (solubility) line. 31 outlying points ( 8 G points outside the gel sphere and $23 \mathbf{P}$ points inside the gel sphere) are created with this method. [Solubility sphere: $\delta_{d}=17.57 ; \delta_{p}=4.89 ; \delta_{h}=13.29 ; R_{\text {sol }}=5.0 \mathrm{MPa}^{1 / 2}$, Gelation sphere: $\mathrm{R}_{\mathrm{Gel}}=12.5 \mathrm{MPa}^{1 / 2}$ ]
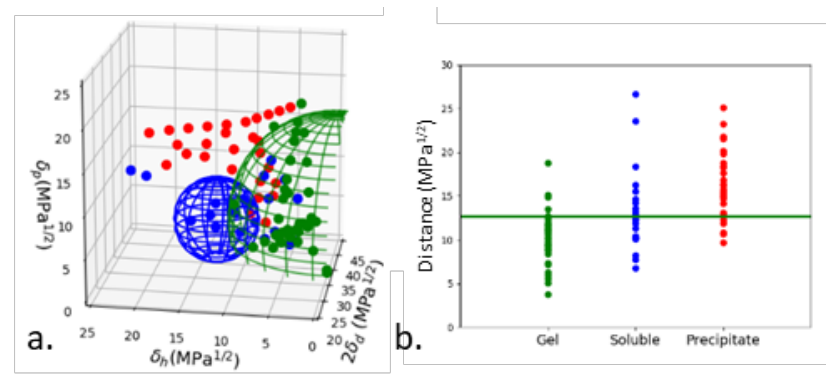

Figure 2. a) Same data as in Fig. 1. The solubility and gelation spheres are not forced to have the same centres (NO method). b) Distances in HSP space of the liquids tested to the centre of the gelation sphere, highlighting the radius of the gelation sphere by a green line. 9 outlying points ( $4 \mathrm{G}$ points outside the gel sphere and $5 \mathbf{P}$ points inside the gel sphere) are created with this method. [Gelation sphere: $\delta_{d}=20.57 ; \delta_{p}=3.71 ; \delta_{h}=$ $\left.0.00 ; \mathrm{R}_{\mathrm{Gel}}=12.7 \mathrm{MPa}^{1 / 2}\right]$

The determination of a gelation sphere for $\mathbf{C} 4$ that has the same centre as the solubility sphere results in a poor fit to the experimental data, with $23 \mathbf{P}$ points and $8 \mathbf{G}$ points as outliers. In contrast, if the centre of the gelation sphere is not imposed to be the same as the centre of the solubility sphere, a better fit is obtained with only $4 \mathbf{P}$ points and $5 \mathbf{G}$ points as outliers (Fig. 2).

\section{Do gelation and solubility spheres overlap?}

Although force fitting the centre of the gelation sphere may not be the best general method for domain determination, we cannot discard the hypothesis that some LMWGs could show a better fit of the data if the gelation and solubility spheres are allowed to overlap (fully or partially). This was actually observed by several authors, 30,32,33,37,38 and we investigate this issue with the following example: LMWG A5, a bisamide based LMWG40 (Fig. 3a) was synthesized and its gelation ability was tested.

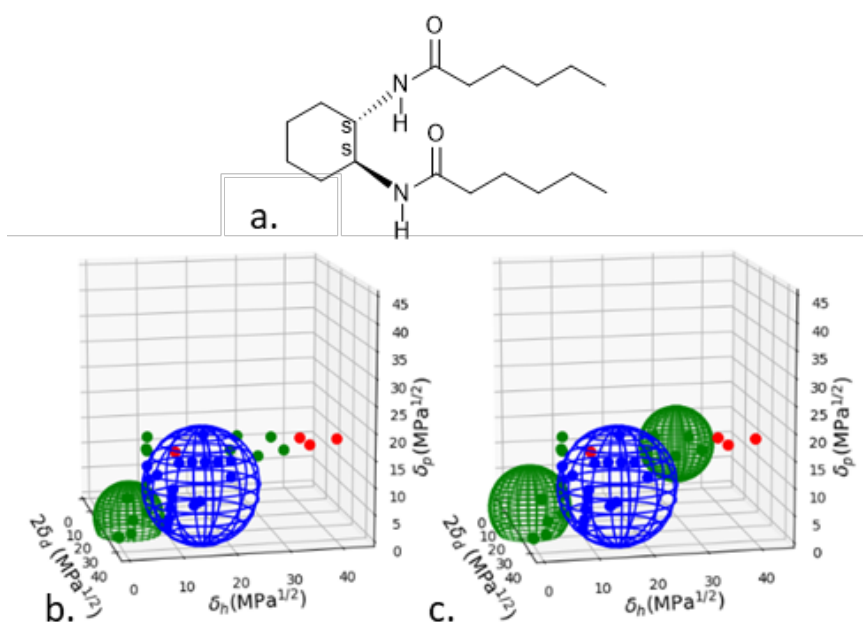

Figure 3. a) LMWG $\mathbf{A} 5$ b) Data for $\mathbf{A} 5$ at $1 \mathrm{wt} / \mathrm{v} \%$ with a single gelation sphere. Liquids are represented by full circles and calculated domains are represented by meshed spheres. Green: gel; Red: precipitate. Blue: soluble. The spheres are determined according to the NO method [Gelation sphere: $\delta_{d}=15.93 ; \delta_{p}=3.33 ; \delta_{h}=2.31 ; R_{G e l}=$ 6.5 $\mathrm{MPa}^{1 / 2}$ ] c) Same data with two gelation spheres. [Gelation sphere 1: $\delta_{d}=15.41 ; \delta_{\mathrm{p}}=$ $5.24 ; \delta_{\mathrm{h}}=0.0 ; \mathrm{R}_{\mathrm{Gel}}=7.5 \mathrm{MPa}^{1 / 2}$, Gelation sphere $2: \delta_{\mathrm{d}}=16.34 ; \delta_{\mathrm{p}}=16.11 ; \delta_{\mathrm{h}}=26.99$; $R_{G e l}=6.8 \mathrm{MPa}^{1 / 2}$ ]. [Solubility sphere: $\delta_{d}=18.56 ; \delta_{p}=9.82 ; \delta_{h}=15.08 ; R_{S o l}=10.9 \mathrm{MPa}^{1 / 2}$ ]

Using our original NO methodology in which the gelation and solubility spheres are not allowed to overlap, we could not obtain a good fit to the experimental data (Fig. 3b). This procedure resulted in $8 \mathbf{G}$ points as outliers. As initially proposed, ${ }^{10}$ this bad fit drove us to test whether the use of two gelation spheres would be more suitable. Of course, this approach results in a better fit showing only $3 \mathbf{G}$ points as outliers (Fig. 3c).

However, a possible reason why two gelation spheres might exist, is if each one is associated to a specific crystalline packing. ${ }^{41,42}$ Therefore, $\mathrm{X}$-ray measures (fig. 4) were performed on xerogels obtained from different liquids in order to test this point. 


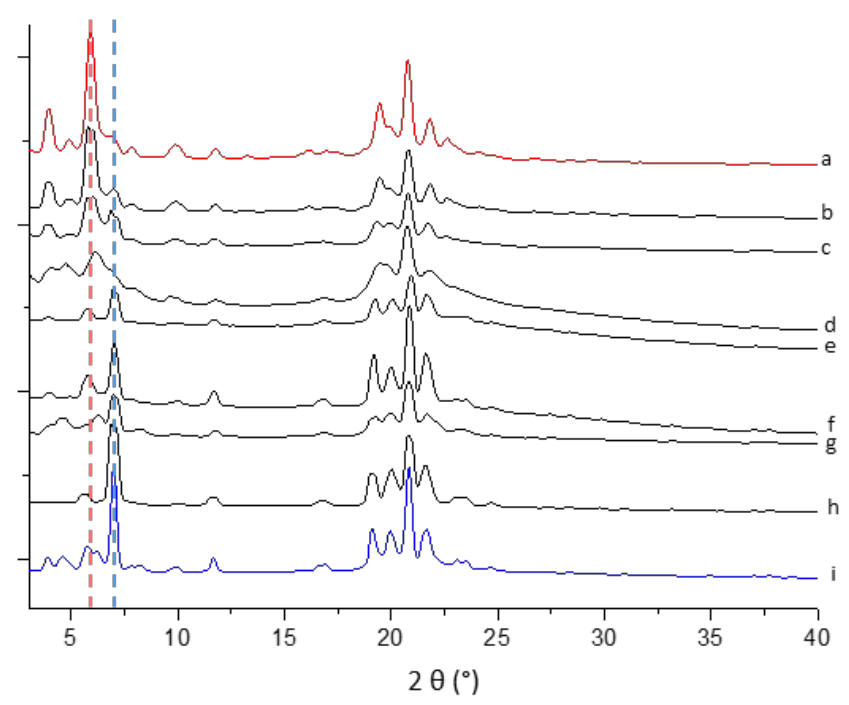

Figure 4. X-ray patterns of xerogels of A5, a - toluene, b - t-butylacetate, c - 1chloropentane, d - acetonitrile, e - cyclohexane, f - chlorobenzene, g methylethylketone, $\mathrm{h}$ - methanol/water (composition:75/25), i - methanol/water (composition:50/50). Diffractograms a to $g$ (respectively $h$ to i) correspond to gels included in the low $\delta_{H}$ (respectively high $\delta_{H}$ ) gelation sphere of Fig. 3.

Indeed, significant differences in the patterns indicate that more than one phase is present. We propose that two phases coexist, one of them being prominent in the xerogel formed in toluene (Fig. 4a) while the other one is prominent in the xerogel obtained from the methanol water mixture (50:50) (Fig. 4i). A precise indexation of both phases is still under investigation, but it is sufficient to notice at this point that the coexistence is observed for both spheres. It means that this polymorphism is not a discriminant factor between the two putative gelation spheres (Fig. 3c), thus not supporting the existence of more than one gelation sphere.

Rogers and Weiss ${ }^{30,37}$ presented an alteration of Weiss original CS methodology 35,36 to determine the gelation sphere where they showed the gelation and solubility spheres sharing a common volume in the Hansen space, without sharing the same centre. Following this idea, we modify our initial methodology to allow overlapping gelation and solubility spheres (AO method stands for Allowed Overlap). We propose to calculate the gelation sphere so that most $\mathbf{G}$ points lie inside the sphere and most $\mathbf{P}$ points lie outside, with no consideration of where the $\mathbf{S}$ points are placed. When this new AO methodology is applied to the data of A5, a large gelation sphere is obtained that encloses the solubility sphere (Fig. 5). It results in a good description of the experimental data with only a single outlier (Fig.6).

When this new AO method is applied to LMWGs'10 that we had previously studied, we obtain similar outcomes as with the NO method (see data in SI). Indeed, these previously studied LMWGs, do not show $\mathbf{G}$ points uniformly distributed around $\mathbf{S}$ points, so that the AO method yields non-overlapping gelation and solubility spheres, with similar centre, radius and number of outliers as the originally proposed NO method. 9,10 Moreover, this $A O$ method was also tested with success on all recent gelation data dealing with the HSP-based rationalization of gelation (see data in $\mathrm{SI}^{16,18,20-23,25-29}$ ).

This indicates that using a methodology that allows but does not force the gelation sphere to share a common volume with the solubility sphere is the most suitable option for a general method for the determination of the gelation domain.

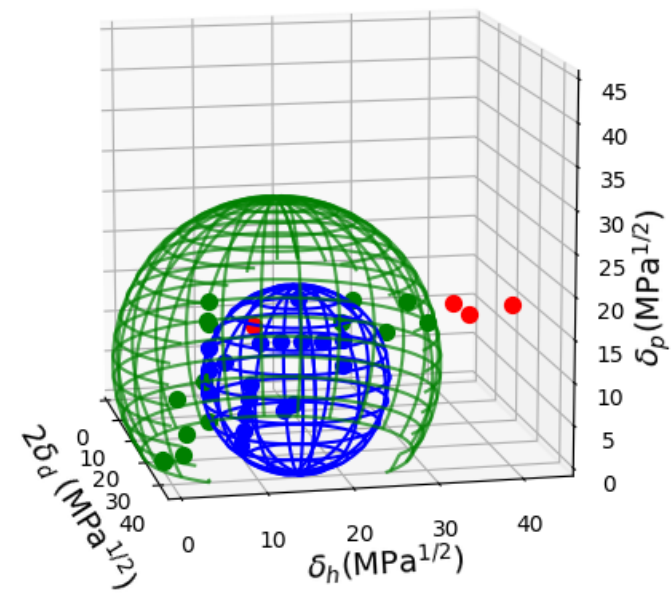

Figure 5 . Same data as in Fig. 3. Liquids are represented by full circles and calculated domains are represented by meshed spheres (AO method). Blue: soluble; Green: gel; Red: precipitate. [Gelation sphere: $\delta_{d}=16.25 ; \delta_{p}=10.94 ; \delta_{h}=13.76 ; R_{G e l}=19.91 \mathrm{MPa}^{1 / 2}$; Solubility sphere: $\left.\delta_{d}=18.56 ; \delta_{p}=9.82 ; \delta_{h}=15.08 ; R_{\text {sol }}=10.9 \mathrm{MPa}^{1 / 2}\right]$

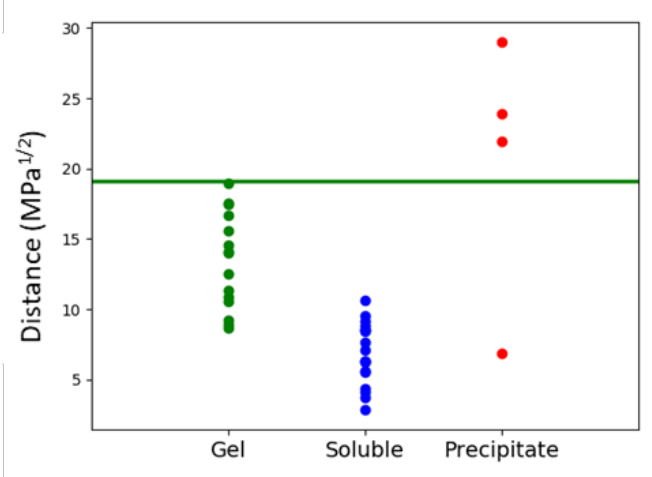

Figure 6. Same data and spheres (AO method) as in Fig. 5. Distances in HSP space of the liquids tested to the centre of the gelation sphere, highlighting the radius of the sphere by a green line. Green: gel; Red: precipitate. Blue: soluble.

\section{The need for a dedicated algorithm}

Finally, it is important to use a precise optimization procedure to determine the centre and radius of the spheres (gelation and solubility). Indeed, the ideal situation where all $\mathbf{G}$ points lie inside the gel sphere and all $\mathbf{P}$ points lie outside is rather rare. Therefore, a quantitative criterion has to be chosen to measure the quality of the fit. Examples of such criterion and minimization algorithm have been proposed by Gharagheizi et $\mathrm{al}^{43}$ or in the HSPiP11-13 or UMD ${ }^{36}$ softwares.

An alternative approach called the minimal enclosing sphere has been proposed. ${ }^{30}$ In this method, the gelation sphere is the smallest sphere that contains all the $\mathbf{G}$ points. The problem with 
this admittedly simple method is that a single $\mathbf{G}$ point that lies far from the other points will considerably and artificially displace the gel sphere.

To illustrate the need of a minimization algorithm we determined the gelation sphere of $\mathbf{C 1 8}^{39}$, using either the minimal enclosing sphere method (Fig. 7) or a dedicated fitting algorithm (Fig. 8). The principle of the algorithm is to minimize the objective function in order to get a sphere which includes a maximum of $\mathbf{G}$ points and a minimum of $\mathbf{P}$ points.

a.
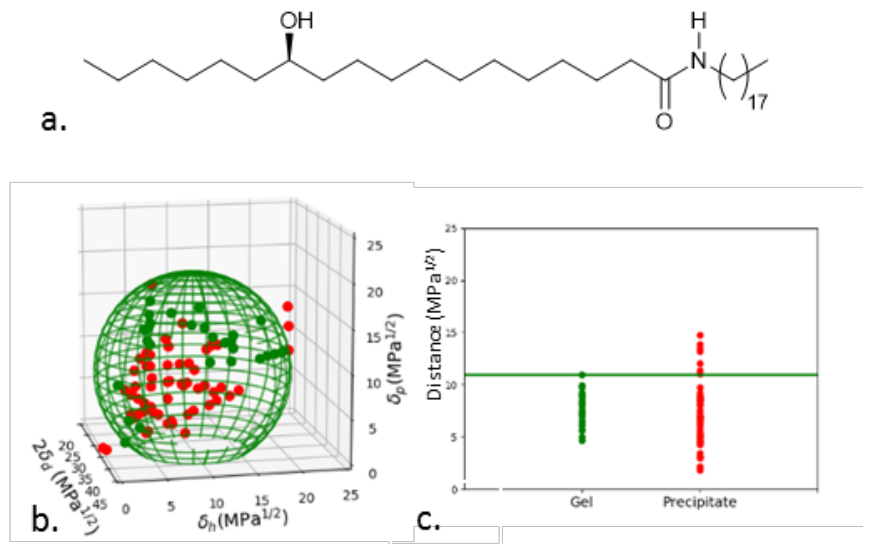

Figure 7. a) LMWG $\mathbf{C} 18$ b) Data for $\mathbf{C} 18$ at $2 \mathrm{wt} / \mathrm{v} \%$ with the gelation sphere calculated using the BoundingRegion function from Wolfram Mathematica 11 (minimal enclosing sphere). Green: gel; Red: precipitate. Liquids are represented by full circles and the gelation sphere is represented by a meshed sphere. The present dataset does not include any S points. [Gelation sphere: $\delta_{d}=18.19 ; \delta_{p}=9.25 ; \delta_{h}=9.62 ; R_{G e l}=10.95 \mathrm{MPa}^{1 / 2}$ ] c) Distances in HSP space of the liquids tested to the centre of the gelation sphere, highlighting the radius of the sphere by a green line. 54 outlying points ( $\mathbf{P}$ points inside the gel sphere) are created with this method (i.e. $60 \%$ of the data).

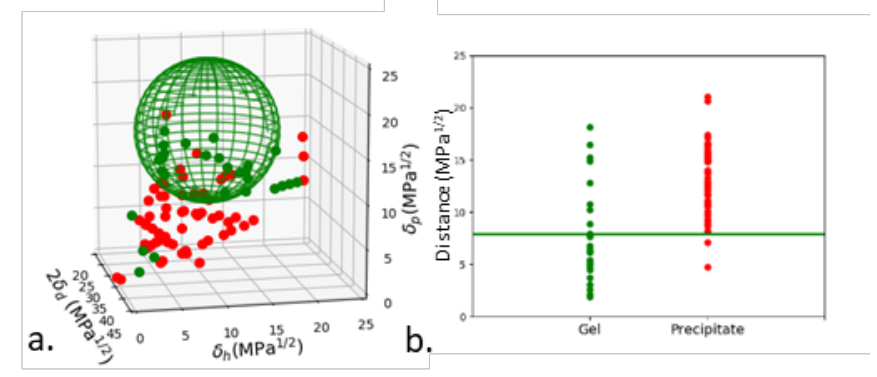

Figure 8. Same data as in Fig. 7. a) The gelation sphere was calculated using the HSPiP software. Green: gel; Red: precipitate. [Gelation sphere: $\delta_{d}=19.65 ; \delta_{p}=9.05 ; \delta_{h}=17.76$; $\mathrm{R}_{\mathrm{Gel}}=7.9 \mathrm{MPa}^{1 / 2}$ ] b) Distances in HSP space of the liquids tested to the centre of the gelation sphere, highlighting the radius of the sphere by a green line. 10 outlying points ( 8 G points outside the gel sphere and $2 \mathbf{P}$ points inside the gel sphere) are created with this method (i.e. $10 \%$ of the data).

In this case the comparison is clear, showing that the minimal enclosing sphere resulted on an overall of 54 outliers compared with only 10 outliers when using the dedicated fitting algorithm.

\section{Experimental}

\section{Synthesis}

Bisamide A5 was synthesized as previously reported. ${ }^{40}$

\section{Gel preparation}

Samples were prepared by adding 10mg of LMWG A5 and $1 \mathrm{~mL}$ of liquid in a screw-cap vial. The suspensions were heated until dissolution and left to cool to room temperature on the bench. After 24 hours the vials were inverted, and the aspect of the samples was noted as gel (G), solution (S) or precipitate (P). A material is considered a gel if no deformation is observed after inverting the vial. Data of LMWGs C4 and C18 were previously described. ${ }^{39}$

\section{Sphere determination}

All solubility and gelation spheres with the exception of the minimal enclosing gelation sphere were calculated using the generic algorithm provided in the HSPiP software. ${ }^{11,12}$ The solubility sphere was calculated in a way that as many $\mathbf{S}$ points as possible lie inside the sphere, but as many $\mathbf{G}$ and $\mathbf{P}$ points as possible lie outside. The gelation sphere determined according to the NO methodology contained as many $\mathbf{G}$ points as possible inside the sphere, but as many $\mathbf{S}$ and $\mathbf{P}$ points as possible outside. Following the new AO methodology, the gelation sphere was determined so that as many $\mathbf{G}$ points as possible lie inside the sphere, but as many $\mathbf{P}$ points as possible lie outside. In other words, the $\mathbf{S}$ points are not taken in consideration for the gelation sphere determination in the AO methodology.

Minimal enclosing sphere was computed using the BoundingRegion formula from Wolfram Mathematica 11.

\section{Data representation}

All data visualization was done with resource to the SciPy Python library. An executable version of our data plot method with easy-to-use features to users can be requested upon demand.

\section{X-ray scattering analysis}

Gel samples were dried under vacuum in order to obtain xerogels. All xerogels were measured in sodaglass capillaries with $1 \mathrm{~mm}$ diameter. The two-dimensional wide-angle X-ray scattering (WAXS) patterns were collected on a MAR345 detector using $\mathrm{Cu}-\mathrm{Ka}$ radiation (wavelength: $1.542 \mathrm{~A}$ ) of a rotating anode $\mathrm{X}$-ray source $(40 \mathrm{kV}, 40 \mathrm{~mA}$; multilayer graded monochromator). Exposure time was 1200s.

\section{Conclusions}

By modifying our previous methodology we have been able to develop a general way of determining the gelation domain of 
LMWGs that is compatible with all the published data. The exclusion of $\mathbf{S}$ points from the gelation sphere determination allows (but does not force) gelation and solubility spheres to share a common volume. This AO methodology yields a good fit to the data, whether the experimental $\mathbf{G}$ and $\mathbf{S}$ points lie in disjoined or imbricated regions. The use of a dedicated algorithm is also necessary for a general methodology since some LMWGs have complex Hansen profiles and a minimal enclosing sphere of the $\mathbf{G}$ points is not robust enough. By presenting a general method for the gelation sphere determination we hope that future comparison between studies that correlate gelation data with HSP will become easier. This would then allow testing possible relationships ${ }^{39}$ between the structure and the gel formation ability of LMWGs.

\section{Conflicts of interest}

There are no conflicts of interest to declare.

\section{Acknowledgements}

This work was supported by the French Agence Nationale de la Recherche (project ANR-16-CE08-0012 HSPgel).

\section{Notes and references}

1 P. Terech and R. G. Weiss, Chem. Rev., 1997, 97, 3133-3160.

2 O. Gronwald, E. Snip and S. Shinkai, Curr. Opin. Colloid Interface Sci., 2002, 7, 148.

3 N. M. Sangeetha and U. Maitra, Chem. Soc. Rev., 2005, 34, 821.

4 M. George and R. G. Weiss, Acc. Chem. Res., 2006, 39, 489.

5 S. Banerjee, R. K. Das and U. Maitra, J. Mater. Chem., 2009, 19, 6649.

6 R. G. Weiss, J. Am. Chem. Soc., 2014, 136, 7519.

7 Y. Ohsedo, Polym. Adv. Technol., 2016, 27, 704.

8 S. Ghosh, V. K. Praveen and A. Ajayaghosh, Annu. Rev. Mater. Res., 2016, 46, 235.

9 M. Raynal and L. Bouteiller, Chem. Commun., 2011, 47, 8271.

10 J. Bonnet, G. Suissa, M. Raynal and L. Bouteiller, Soft Matter, 2014, 10, 3154.

11 C. M. Hansen, HANSEN SOLUBILITY PARAMETERS A User' 's Handbook Second Edition, 2007.

12 S. Abbott and C. M. Hansen, Hansen Solubility Parameters in Practice, 2008.

13 M. J. Louwerse, A. Maldonado, S. Rousseau, C. MoreauMasselon, B. Roux and G. Rothenberg, ChemPhysChem, 2017, 18, 2999.

14 Y. Lan, M. G. Corradini, R. G. Weiss, S. R. Raghavan and M. A. Rogers, Chem. Soc. Rev., 2015, 44, 6035.

15 J. Gao, S. Wu and M. A. Rogers, J. Mater. Chem., 2012, 22, 12651.

16 H. Xu, J. Song, T. Tian and R. Feng, Soft Matter, 2012, 8, 3478.

17 F. Aparicio, F. García and L. Sánchez, Chem. - A Eur. J., 2013, 19, 3239.

18 W. Edwards and D. K. Smith, J. Am. Chem. Soc., 2013, 135, 5911.
19 S. Wu, J. Gao, T. J. Emge and M. A. Rogers, Soft Matter, 2013, 9, 5942.

20 V. C. Edelsztein, A. S. Mac Cormack, M. Ciarlantini and P. H. Di Chenna, Beilstein J. Org. Chem., 2013, 9, 1826.

21 T. Ando and K. Ito, J. Incl. Phenom. Macrocycl. Chem., 2014, 80, 285.

22 S. He, H. Zhao, X. Guo, X. Xu, X. Zhou, J. Liu, Z. Xing, L. Ye, L. Jiang, Q. Chen and Y. He, Chem. - A Eur. J., 2014, 20, 15473.

23 C. Tong, K. Fan, L. Niu, J. Li, X. Guan, N. Tao, H. Shen and J. Song, Soft Matter, 2014, 10, 767.

24 H. Shen, L. Niu, K. Fan, J. Li, X. Guan and J. Song, Langmuir, 2014, 30, 9176.

25 Y. Huang, Y. Yuan, W. Tu, Y. Zhang, M. Zhang and H. Qu, Tetrahedron, 2015, 71, 3221.

26 Y. Li, X. Ran, Q. Li, Q. Gao and L. Guo, Chem. - An Asian J., 2016, 11, 2157.

27 T. L. Lai, D. Canevet, N. Avarvari and M. Sallé, Chem. - An Asian J., 2016, 11, 81.

28 T. Wang, X. Yu, Y. Li, J. Ren and X. Zhen, ACS Appl. Mater. Interfaces, 2017, 9, 13666.

29 T. Xiao, X. Zhang, J. Wu, J. Yang and Y. Yang, Chempluschem, 2017, 82, 879

30 Y. Lan, M. G. Corradini, X. Liu, T. E. May, F. Borondics, R. G. Weiss and M. A. Rogers, Langmuir, 2014, 30, 14128.

31 Y. Yin, Z. Gao, Y. Bao, B. Hou, H. Hao, D. Liu and Y. Wang, Ind. Eng. Chem. Res., 2014, 53, 1286.

32 M. Zhang, S. Selvakumar, X. Zhang, M. P. Sibi and R. G. Weiss, Chem. - A Eur. J., 2015, 21, 8530.

33 C. Liu, M. Corradini and M. A. Rogers, Colloid Polym. Sci., 2015, 293, 975.

34 N. Yan, Z. Xu, K. K. Diehn, S. R. Raghavan, Y. Fang and R. G. Weiss, Langmuir, 2013, 29, 793.

35 N. Yan, Z. Xu, K. K. Diehn, S. R. Raghavan, Y. Fang and R. G. Weiss, J. Am. Chem. Soc., 2013, 135, 8989.

36 K. K. Diehn, H. Oh, R. Hashemipour, R. G. Weiss and S. R. Raghavan, Soft Matter, 2014, 10, 2632.

37 Y. Lan, M. G. Corradini and M. A. Rogers, Cryst. Growth Des., 2014, 14, 4811.

38 A. Singh, F.-I. I. Auzanneau, M. G. Corradini, G. Grover, R. G. Weiss and M. A. Rogers, Langmuir, 2017, 33, 10907.

39 J. Bonnet, G. Suissa, M. Raynal and L. Bouteiller, Soft Matter, 2015, 11, 2308.

40 N. Zweep, A. Hopkinson, A. Meetsma, W. R. Browne, B. L. Feringa and J. H. Van Esch, Langmuir, 2009, 25, 8802.

41 J. Gao, S. Wu, T. J. Emge and M. A. Rogers, CrystEngComm, 2013, 15, 4507.

42 C. Zhao, H. Wang, B. Bai, S. Qu, J. Song, X. Ran, Y. Zhang and M. Li, New J. Chem., 2013, 37, 1454.

43 F. Gharagheizi, J. Appl. Polym. Sci., 2007, 103, 31. 